\title{
Avaliação da qualidade de vida e da perda urinária de mulheres com bexiga hiperativa tratadas com eletroestimulação transvaginal ou do nervo tibial
}

\author{
Evaluation of quality of life and loss urine of women with overactive bladder treated with \\ intravaginal or tibial nerve electro stimulation
}

Maíra de Menezes Franco ${ }^{1}$, Flaviane de Oliveira Souza ${ }^{2}$, Elaine Cristine Lemes Mateus de Vasconcelos ${ }^{3}$, Maurício Mesquita Sabino de Freitas ${ }^{4}$, Cristine Homsi Jorge Ferreira ${ }^{5}$

Estudo desenvolvido na Faculdade de Medicina de Ribeirão Preto da USP Universidade de São Paulo - Ribeirão Preto (SP), Brasil.

1 Mestranda do Departamento de Biomecânica, Medicina e Reabilitação do Aparelho Locomotor da Faculdade de Medicina de Ribeirão Preto da Universidade de São Paulo (USP) Ribeirão Preto (SP), Brasil.

2 Fisioterapeuta; Mestre pelo Departamento de Biomecânica, Medicina e Reabilitação do Aparelho Locomotor, da Faculdade de Medicina de Ribeirão Preto da USP Ribeirão Preto (SP), Brasil.

${ }^{3}$ Fisioterapeuta do Hospital das Clínicas da Faculdade de Medicina de Ribeirão Preto da USP - Ribeirão Preto (SP), Brasil.

${ }^{4}$ Docente do Departamento de Ginecologia e Obstetrícia da Faculdade de Medicina de Ribeirão Preto da USP - Ribeirão Preto (SP), Brasil.

${ }^{5}$ Docente do Departamento de Biomecânica, Medicina e Reabilitação do Aparelho Locomotor da Faculdade de Medicina de Ribeirão Preto da USP Ribeirão Preto (SP), Brasil.

ENDEREÇO PARA CORRESPONDÊNCIA:

Maíra de Menezes Franco - Av.

Governador Lucas Nogueira Garcez, 70

- Parque Residencial Cidade Universitária

- CEP: 14051-510 - Ribeirão Preto (SP),

Brasil - E-mail: mairamfranco@yahoo.com

APRESENTACÃA:

maio 2010

ACEITO PARA PUBLICAÇÃO: jan. 2011

FONTE DE FINANCIAMENTO: nenhuma

CONFLITO DE INTERESSE: nada a declarar

Este estudo teve apoio da empresa Quark Produtos Médicos.
RESUMO: Trata-se de um ensaio clínico prospectivo comparativo que objetivou comparar os efeitos do tratamento com eletroestimulação transvaginal (ET) e do nervo tibial (ENT) sobre a qualidade de vida (QV) e queixas de perda urinária em mulheres com bexiga hiperativa. Participaram 42 pacientes com bexiga hiperativa ou incontinência urinária (IU) mista e foram divididas para tratamento com ET ou ENT. A QV foi avaliada pelo questionário de QV genérico, o Medical Outcomes Study Short Form 36 (SF-36) e um questionário específico para IU, o Incontinence Quality of Life Instrument (I-QOL). Os relatos de perdas urinárias e incômodos ocasionados foram avaliados, respectivamente, por meio do diário miccional de 24 horas e Escala Visual Analógica (EVA). O tratamento foi realizado uma vez por semana, totalizando doze semanas. O grupo da ENT teve melhora significativa em três domínios do I-QOL, na EVA, que avaliou o grau de incômodo causado pela IU e em quatro aspectos do diário miccional. No grupo de ET houve melhora significativa de dois domínios do SF-36, três domínios do I-QOL, na EVA e em quatro aspectos do diário. Houve melhora da QV em ambos os grupos, assim como uma diminuição das queixas de perda urinária, entretanto, o grupo que recebeu ET obteve melhora nos escores em dois domínios do questionário de QV genérico após o tratamento, que teve limitação por aspectos físicos e limitação por aspectos emocionais. O que não ocorreu com o grupo de ENT.

Descritores: fisioterapia; bexiga urinária hiperativa; estimulação elétrica; qualidade de vida; nervo tibial posterior.

ABSTRACT: This is a prospective comparative clinical study. This study aimed to compare the effects of intravaginal electrical stimulation therapy (IS) and the tibial nerve (TNS) on quality of life (QOL) and complaints of urinary incontinence in women with overactive bladder. Participants 42 patients with overactive bladder or urinary incontinence (UI) mix and were divided for treatment with IS or TNS. To assess QOL we used a generic QOL questionnaire, the Medical Outcomes Study Short Form 36 (SF-36) and a specific questionnaire for urinary incontinence, the Incontinence Quality of Life Instrument (I-QOL). The reports of urinary incontinence and discomfort caused were evaluated, respectively, through the 24-hour voiding diary and visual analog scale (VAS). Treatment was performed once a week, twelve weeks in total. The PTNS group had significant improvement in three areas of the I-QOL in VAS, which assessed the degree of discomfort caused by IU and four aspects of voiding diary. In the IS group significantly improved in two domains of the SF-36 domains of the three $\mathrm{I}-\mathrm{QOL}$ in VAS and on four issues of the journal. There was improvement in QOL in both groups, as well as a decrease in complaints of urinary leakage, however, the group that received IS had been improved scores in two areas of the generic QOL questionnaire after treatment, which were limited by the physical limitation and by emotional aspects. What did not happen with the group of TNS.

KeYwords: physiotherapy; overactive detrusor; electric stimulation; quality of life; tibial nerve. 


\section{INTRODUCÃO}

Segundo a Sociedade Internacional de Continência (ICS) a incontinência urinária (IU) é a queixa de qualquer perda involuntária de urina ${ }^{1}$. Constitui uma afecção que não configura risco de vida, mas causa profundas mudanças psicossociais à mulher ${ }^{2}$. Compromete $\mathrm{a}$ vida social, psicológica, doméstica, ocupacional, física e sexual de 15 a 30\% das mulheres de todas as idades. As repercussões ocasionadas pela IU pode ainda desencadear perda da autoconfiança, da auto-estima, levando-as a desenvolverem quadros de angústia e depressão ${ }^{3}$.

A bexiga hiperativa $(\mathrm{BH})$ é, depois da IUE, a desordem do trato urinário inferior mais frequente observada nas mulheres e seus sintomas incluem urgência miccional e/ou urge-incontinência, polaciúria e noctúria ${ }^{1,4}$. A literatura internacional ${ }^{5-7}$ e nacional ${ }^{8,9}$ apontam maior repercussão negativa sobre a qualidade de vida (QV) nos casos de urge-incontinência do que nos outros tipos de IU.

A eletroestimulação é uma modalidade fisioterapêutica utilizada no tratamento da $\mathrm{BH}$. Basicamente, seu objetivo é inibir a contração involuntária do detrusor $^{10}$. Diversos estudos ${ }^{3,11,12}$ verificaram boa efetividade no tratamento da $\mathrm{BH}$ com a utilização da eletroestimulação transvaginal (ET). Pesquisas mostram que baixas freqüências, de 5 a 10 Hertz (Hz), são ótimas para a inibição central dos neurônios motores parassimpáticos, promovendo resultados que variam extensamente, com 45 a $91 \%$ de efetividade ${ }^{13,14}$.

Mais recentemente, a eletroestimulação do nervo tibial (ENT) no tratamento da $\mathrm{BH}$ vem sendo estudada e discutida com eletrodos de inserção (subcutâneos) e superficiais, demonstrando resultados favoráveis, constituindo-se uma opção nos casos de contraindicação ou intolerância à $E T^{15-17}$. Entretanto, a repercussão da ENT sobre a QV de mulheres com BH tem sido pouco estudada e parece não haver estudos que comparem a repercussão desse tratamento com o tratamento de ET. Assim, os objetivos desta pesquisa foram avaliar e comparar os efeitos da ET ou ENT sobre a QV de mulheres com IU, avaliar sua repercussão na queixa clínica de $\mathrm{BH}$ e IU mista e verificar o grau de satisfação destas pacientes com o tratamento fisioterapêutico proposto.

\section{MATERIAL E MÉTODOS}

Trata-se de um ensaio clínico prospectivo comparativo. Foi desenvolvido na Seção de Fisioterapia e no Ambulatório de Uroginecologia, Cirurgia Ginecológica e Cirurgia Reconstrutiva Pélvica do Hospital das Clínicas da Faculdade de Medicina de Ribeirão Preto da Universidade de São Paulo (HCFMRP-USP). Teve aprovação pelo Comitê de Ética em Pesquisa da mesma instituição, em 04/12/2006 com processo $n^{\circ} .13247 / 2006$.

O cálculo amostral para a variável Incontinence Quality of Life Instrument (I-QOL), foi obtido após realização de estudo piloto, estimando um número de 40 pacientes.

As pacientes foram divididas em: grupo de ET (grupo A) com 20 pacientes ou grupo de ENT (Grupo B) com 22. A divisão nos grupos foi feita alternadamente seguindo a ordem que a paciente era avaliada e aceitava entrar para a pesquisa.

Todas as pacientes incluídas no estudo aceitaram participar mediante esclarecimentos e assinatura do termo de consentimento livre e esclarecido. Eram alfabetizadas e tiveram diagnóstico de $\mathrm{BH}$ ou IU mista segundo estudo urodinâmico. Não faziam uso de medicação para $\mathrm{BH}$ ou faziam uso da medicação há mais de três meses. Foram excluídas: grávidas ou no período de lactação, que tiveram infecção urinária ou vaginal, distopias vaginais grau III, portadoras de tumores intrapélvicos, que faziam uso de dispositivo intrauterino (DIU), que tinham marcapasso cardíaco, problemas cognitivos, desordens neurológicas, cardiopatias ou implantes metálicos na região do quadril e que interromperam uso de medicação que faziam uso durante a coleta dos dados.

Para avaliação da QV, foi utilizado um questionário de QV genérico, o Medical Outcomes Study Short Form 36 (SF-36), instrumento validado em português por Ciconelli ${ }^{18}$, e um questionário de QV específico para IU, o I-QOL ${ }^{19}$. Ambos foram aplicados antes e após as 12 sessões de tratamento.

Para avaliação das queixas clínicas das pacientes com $\mathrm{BH}$ e IU mista foi utilizado o diário miccional de 24 horas $^{20}$ e para estimar o grau de incômodo da perda urinária foi utilizada a escala visual analógica (EVA) ${ }^{21}$. Para verificar o grau de satisfação destas pacientes com o tratamento fisioterapêutico proposto, foi realizada uma avaliação subjetiva em relação às queixas clínicas e satisfação com o tratamento, na qual a voluntária relatou se considerava estar: curada, melhor e satisfeita com o tratamento (não solicitando alternativa terapêutica), melhor e insatisfeita com o tratamento (solicitando alternativa terapêutica), ou inalterada ${ }^{22}$.

As pacientes foram submetidas a 12 sessões de ET ou ENT, sendo uma sessão semanal e individual. Para ambos os tipos de eletroestimulação foi utilizado o equipamento Dualpex 961 da Quark ${ }^{\circledR}$. Os parâmetros de ET adotados foram frequência de $10 \mathrm{~Hz}$, largura de pulso de $700 \mu$ s e tempo de tratamento de 20 minutos por sessão. Para a ENT foram utilizados frequência de $20 \mathrm{~Hz}$, largura de pulso de $200 \mu$ s e tempo de 30 minutos por sessão. Ambos os parâmetros com intensidades de acordo com a tolerância da paciente. Um par de eletrodos de silicone era posicionado, sendo um eletrodo acima do maléolo medial esquerdo e outro abaixo do mesmo, próximo ao arco plantar do pé esquerdo, de acordo com estudo feito por Amarenco et $\mathrm{al}^{17}$.

$\mathrm{Na}$ análise estatística foi verificada a associação entre as variáveis qualitativas, realizada análise descritiva, utilizado teste exato de Fisher, teste $t$ de Student e modelo linear de efeitos mistos. Para todos os cálculos foi utilizado o software SAS versão 9 .

\section{RESULTADOS}

A Tabela 1 contém a caracterização dos grupos A e B. Não houve diferença estatística entre eles.

Na avaliação da QV pelo SF-36, a comparação prévia ao tratamento entre os grupos apresentou uma diferença significativa em apenas um domínio: vitalidade $(p=0,05)$. As diferenças não 
foram estatisticamente significativas em nenhum domínio quando comparados os dois grupos estudados após o tratamento. A comparação entre o antes de depois de cada grupo encontra-se na Tabela 2.

Avaliando o I-QOL e o do diário miccional, não houve diferença significativa entre os grupos na avaliação inicial e na avaliação final. Nas Tabelas 3 e 4, encontram-se os valores antes e depois da intervenção de cada grupo, do I-QOL e do diário miccional, respectivamente.

Em relação ao grau de incômodo gerado pela IU, avaliado pela EVA, houve diferença significativa antes e depois do tratamento em ambos os grupos (Tabela 4), porém não houve diferença significativa comparando os grupos na avaliação antes do tratamento $(p=0,68)$ e depois do tratamento $(p=0,74)$.

Em relação à avaliação subjetiva, $5 \%$ das mulheres do grupo $A(n=1)$

Tabela 1. Dados demográficos dos Grupos A e B

\begin{tabular}{llccc}
\hline Variável & & Grupo A $\mathbf{n}=\mathbf{2 0}$ & Grupo $\mathbf{B} \mathbf{n}=\mathbf{2 2}$ & Valor $\mathbf{p}$ \\
\hline Idade (anos) & $50,25 \pm 13,04$ & $49,32 \pm 14,75$ & 0,76 \\
IMC kg $\left(\mathrm{m}^{2}\right)$ & $32,55 \pm 6,3$ & $30,45 \pm 6,55$ & 0,14 \\
Tipo de IU & Hiperatividade do detrusor & 13 & 14 & 0,99 \\
& Incontinencia mista & 7 & 8 & 0,46 \\
Tabagismo & Sim & 4 & 6 & 16 \\
& Não & 16 & \\
\hline
\end{tabular}

Tabela 2. Avaliação da Qualidade de Vida SF-36 antes e após o tratamento

\begin{tabular}{|c|c|c|c|c|c|c|}
\hline \multirow[t]{2}{*}{ Domínio } & \multicolumn{3}{|c|}{ Grupo A $(n=20)$} & \multicolumn{3}{|c|}{ Grupo B $(n=22)$} \\
\hline & Antes & Após & Valor $p$ & Antes & Após & Valor $p$ \\
\hline Capacidade funcional & $57,5 \pm 29,31$ & $62 \pm 32,26$ & 0,35 & $55,45 \pm 32,77$ & $58,64 \pm 29,53$ & 0,49 \\
\hline Aspectos físicos & $28,75 \pm 37,41$ & $57,5 \pm 42,22$ & $0,01^{*}$ & $51,14 \pm 41,89$ & $63,64 \pm 44,14$ & 0,44 \\
\hline Dor & $41,85 \pm 27,5$ & $52,2 \pm 30,35$ & 0,12 & $42,41 \pm 22,25$ & $49,36 \pm 28,91$ & 0,27 \\
\hline Estado saúde & $54,75 \pm 22,58$ & $61 \pm 25,56$ & 0,25 & $50,93 \pm 23,5$ & $59,32 \pm 25,08$ & 0,11 \\
\hline Vitalidade & $54,5 \pm 23,84$ & $56,5 \pm 24,5$ & 0,73 & $40 \pm 19,7$ & $44,32 \pm 24,65$ & 0,44 \\
\hline Aspectos sociais & $55,63 \pm 27,05$ & $64,38 \pm 36,11$ & 0,21 & $61,37 \pm 28,59$ & $69,33 \pm 30,54$ & 0,23 \\
\hline Aspectos emocionais & $35 \pm 41,15$ & $56,67 \pm 43,39$ & $0,01^{*}$ & $43,94 \pm 39,02$ & $66,67 \pm 42,41$ & 0,07 \\
\hline Saúde mental & $56,8 \pm 24,3$ & $60 \pm 24,76$ & 0,54 & $50,73 \pm 22,16$ & $46,36 \pm 22,58$ & 0,38 \\
\hline
\end{tabular}

*Diferença significativa

Tabela 3. Avaliação da Qualidade de Vida I-QOL comparando antes e após o tratamento de cada grupo

\begin{tabular}{lcccccc}
\hline & \multicolumn{3}{c}{ Grupo A $(\mathbf{n}=\mathbf{2 0})$} & & \multicolumn{2}{c}{ Grupo B $(\mathbf{n}=22)$} \\
Domínio & Antes & Após & Valor $\mathbf{p}$ & Antes & Após & Valor $\mathbf{p}$ \\
\hline Limitação do & $51,56 \pm 27,81$ & $64,31 \pm 23,11$ & $0,02^{*}$ & $42,61 \pm 26,4$ & $64,06 \pm 30,23$ & $<0,01^{*}$ \\
comportamento & & & & & & \\
Impacto psicossocial & $48,75 \pm 31,47$ & $67,83 \pm 25,75$ & $<0,01^{*}$ & $56,31 \pm 25,79$ & $70,58 \pm 31,14$ & $<0,01^{*}$ \\
Aspecto social & $37 \pm 31,51$ & $55,53 \pm 31,35$ & $<0,01^{*}$ & $33,41 \pm 26,02$ & $56,59 \pm 32,64$ & $<0,01^{*}$ \\
Total & $47,1 \pm 28,43$ & $63,76 \pm 23,92$ & $<0,01^{*}$ & $46,26 \pm 24,37$ & $65,24 \pm 29,76$ & $<0,01^{*}$ \\
\hline
\end{tabular}

*Diferença significativa

Tabela 4. Diário Miccional comparando antes e após o tratamento de cada grupo

\begin{tabular}{|c|c|c|c|c|c|c|c|}
\hline \multirow[t]{2}{*}{ EVA } & & \multicolumn{3}{|c|}{ Grupo A $(n=20)$} & \multicolumn{3}{|c|}{ Grupo B $(n=22)$} \\
\hline & & Antes & Após & Valor $p$ & Antes & Após & Valor $p$ \\
\hline \multirow{6}{*}{$\begin{array}{l}\text { Diário } \\
\text { miccional }\end{array}$} & & $7,39 \pm 3,12$ & $4,75 \pm 3,62$ & $<0,01 *$ & $7 \pm 2,84$ & $5,11 \pm 3,46$ & $0.03 *$ \\
\hline & Micções & $11,84 \pm 4,29$ & $9,76 \pm 2,49$ & $0,04^{*}$ & $11,68 \pm 4,12$ & $9 \pm 2,71$ & $<0,01^{*}$ \\
\hline & Noctúria & $2,05 \pm 1,31$ & $0,94 \pm 0,9$ & $<0,01 *$ & $1,58 \pm 1,12$ & $1,16 \pm 0,9$ & $0,04^{*}$ \\
\hline & Tempo (min.) & $56,84 \pm 40,15$ & $82,06 \pm 48,58$ & $0,04 *$ & $56,84 \pm 40,63$ & $75 \pm 43,62$ & $0,04 *$ \\
\hline & Perdas & $6,89 \pm 6,69$ & $4,06 \pm 4,51$ & $0,01 *$ & $4,79 \pm 4,97$ & $2 \pm 3,76$ & $<0,01^{*}$ \\
\hline & Absorventes & $1,42 \pm 1,92$ & $1,47 \pm 1,87$ & 0,89 & $1,47 \pm 1,98$ & $1,11 \pm 2,62$ & 0,07 \\
\hline
\end{tabular}

*Diferença significativa 
considerou-se curada, $50 \% \quad(n=10)$ melhor e satisfeita, 35\% $(n=7)$ melhor e insatisfeita e $10 \%(n=2)$ inalterada. No grupo B, 4,54\% $(n=1)$ considerou-se curada, 36,36\% $(n=8)$ melhor e satisfeita, 36,36\% $(n=8)$ melhor e insatisfeita e $22,72 \%(n=5)$ inalterada. Não houve diferença estatística entre os grupos $(p=0,76)$.

\section{DISCUSSÃO}

Os resultados deste estudo indicaram que não houve diferença na QV entre os grupos que foram tratados com ET (A) ou ENT (B), entretanto o grupo A apresentou melhora em dois domínios quando comparado antes e depois do tratamento, limitação por aspectos físicos e limitação por aspectos emocionais, o que demonstra que em relação à QV geral, o tratamento com ET evidenciou uma vantagem em relação ao tratamento com ENT. Os grupos foram homogêneos em relação a diversas variáveis que poderiam influenciar nos resultados tais como idade, IMC, tabagismo e diagnóstico de IU.

A percepção externada por $80 \%$ das mulheres do grupo B de que o local onde era realizada a ENT causava-Ihes estranheza, pode ter contribuído para os resultados obtidos. Este estranhamento pode ter levado essas mulheres a questionar a efetividade da terapêutica, uma vez que a mesma não tinha relação com o local onde elas identificavam o "problema". Entretanto, esta observação foi feita por meio do registro de comentários das participantes, merecendo futuras investigações. Apesar dos questionários de QV genéricos abrangerem conceitos de saúde geral e avaliarem aspectos no campo físico, psicológico e social, não são tão sensíveis na detecção de possíveis mudanças relacionadas especificamente à IU que impactam sobre a QV. No entanto, no estudo de Pal et al. ${ }^{23}$, houve melhora significativa em 5 dos 8 domínios do SF-36 depois do tratamento da IU por BH com ENT. Já o estudo de Balken ${ }^{15}$ observou melhora da QV utilizando o mesmo instrumento após tratamento com ENT, mas somente nos domínios de limitação por aspectos físicos e limitação por aspectos sociais. O estudo de Peters et al..$^{24}$ também utilizou a ENT e o SF-36. Houve melhora significativa em dois domínios, limitação por aspectos físicos e limitação por aspectos emocionais. Contudo, a literatura relata que, apesar de questionários gerais de QV, como o SF-36, serem utilizados para avaliar os efeitos do tratamento, os resultados obtidos, podem ser influenciados por diversos fatores, tais como comorbidades, o que reforça a necessidade de se utilizar associadamente um questionário de QV específico para $I U^{5}$.

Em relação ao $\mathrm{I}-\mathrm{QOL}$, ambos os grupos obtiveram melhora significativa em todos os domínios, mostrando que o tratamento foi eficaz em relação ao impacto da IU sobre a QV. O questionário de QV específico reflete melhor a mudança na resposta ao tratamento. O I-QOL apresenta uma boa reprodutibilidade teste-reteste, porém, a sensibilidade a mudanças é pequena ${ }^{25}$, outro fator limitante do estudo é que esse instrumento apesar de ter sido traduzido para o português não foi validado nesta língua. No presente estudo, a diferença antes e depois do tratamento foi significativa, assim como no estudo de Berghmans ${ }^{12}$, que também utilizou o I-QOL para avaliar tratamento com ET na BH e apresentou diferença significativa após tratamento. No estudo de Pal et al. ${ }^{23}$, Svihra et al. ${ }^{16}$ e Vandoninck et al. ${ }^{26}$ foi utilizado I-QOL para avaliar os efeitos do tratamento com ENT e houve melhora significativa no escore total, assim como no presente estudo. Atualmente, a ET é um recurso que vem sendo amplamente utilizado no tratamento da $\mathrm{BH}^{11,12}$. Já a ENT é relativamente nova, mas alguns estudos demonstram sua eficácia. No estudo de Svihra et al. ${ }^{16}$ observaram melhora dos sintomas de $\mathrm{BH}$ em 56\% das pacientes, acompanhada de uma melhora significativa na QV avaliada pelo I-QOL, assim como no presente estudo.

Um número maior de mulheres que receberam a ET relataram melhora subjetiva do problema, entretanto não houve diferença entre os grupos. A EVA evidenciou que o grau de incômodo causado pela IU, relatado pelas pacientes dos dois grupos depois do tratamento, foi significativamente menor do que antes do tratamento. A EVA é um método simples, confiável e reprodutível para avaliar a QV ${ }^{21,27}$.

Na análise do diário miccional, apenas em relação ao número de absorventes utilizados, não houve diferença significativa, comparando antes e depois do tratamento de ambos os grupos. Apesar do diário miccional constituir uma ferramenta importante para avaliar os resultados terapêuticos, existem divergências sobre o seu real valor, bem como a duração ideal ou mesmo do valor discriminatório e da reprodutibilidade das informações obtidas ${ }^{27}$. No estudo de Melick et al. ${ }^{20}$, um percentual elevado de voluntárias avaliadas preencheram o diário erroneamente, assim como aconteceu com algumas voluntárias do presente estudo, que não completaram partes do dia ou da noite ou preencheram de maneira que impossibilitava sua interpretação. Barroso et al. ${ }^{2}$, utilizaram o diário miccional de 24 horas antes e depois do tratamento com ET em mulheres incontinentes e houve redução significativa no número total de micções, nocturia e no número de perdas. No estudo de Pal et al. ${ }^{23}$, houve diferença significativa comparando antes e depois do tratamento com ENT em quatro variáveis do diário miccional, de 24 horas, nocturia, média de volume urinado, número de episódios de perda, número de absorventes usados, contrariamente Peters et al. ${ }^{24}$, verificaram diminuição da nocturia, episódios de urge-incontinência e volume de urina eliminado. No presente estudo houve melhora significativa antes e depois do tratamento em ambos os grupo, em relação ao número de micções, tempo entre uma micção e outra, nocturia e número de perdas urinárias. Mas não houve diferença comparando os grupos estudados.

Acredita-se que o presente estudo tenha contribuído para uma maior compreensão da repercussão de duas modalidades fisioterapêuticas sobre a QV e queixas de IU em mulheres com BH e IU mista, tendo em vista o fato de não ter sido encontrado na literatura mundial estudos que tenham comparado as duas modalidades terapêuticas relacionadas à QV. O conceito de efetividade terapêutica considera cada vez mais a QV e as queixas clínicas como importantes indicadores, 
entretanto, sugere-se futuras pesquisas que avaliem e comparem a efetividade de tais modalidades terapêuticas como variáveis primárias em ensaios clínicos aleatorizados e controlados, assim como as crenças das mulheres tratadas relacionadas à efetividade das duas modalidades terapêuticas em estudos de abordagem qualitativa.

\section{CONCLUSÃO}

Apesar de somente o grupo de ET ter apresentado melhora na QV pelo instrumento genérico, ambos os grupos obtiveram melhora na QV segundo o questionário específico. Houve melhora clínica da IU avaliada por meio do diário miccional e EVA, tanto mediante a ET quanto ENTP.

\section{REFERENNCIAS}

1. Abrams $P$, Cardozo L, Fall M, Griffiths D, Rosier P, Ulmsten U, van Kerrebroeck P, Victor A, Wein A. The stardardisation of termiology of lower urinary tract function: report from the standardisation sub-committee of the international continence society. Neurourol Urodyn. 2002;21:167-78.

2. Barroso JCV, Ramos JGL, Martins-Costa S, Sanches PRS, Muller AF. Transvaginal electrical stimulation in the treatment of urinary incontinence. BJU Int. 2004;93:31923.

3. Fonseca ESM, Fonseca MCM. Incontinência Urinária Feminina e Qualidade de Vida. In: Moreno A, editor. Fisioterapia em Uroginecologia. Barueri: Manole; 2004. p.95-97.

4. Berghmans B, Doorn EW, Nieman F, Bie R, Brandt PD, Kerrebroeck PV. Efficacy of physical therapeutic modalities in women with proven bladder overactivity. Eur Urol. 2002;41:581-587.

5. Paick JS, Kim SW, Oh SJ, Ku JH. A generic health-related quality of life instrument, the Medical Outcomes Study Short Form-36, in women with urinary incontinence. Eur J Obstet Gynecol and Reprod Biol. 2007;130:18-24.

6. Espuña-Pons M, Puig-Clota M. Lower urinary tract symptoms in women and impact on quality of life. Results of the application of the King's Health Questionnaire. Actas Urol Esp. 2006;30(7):684-91.

7. Rivalta M, Sighinolfi MC, De Stefani S, Bianchi G. Sexual Function and Quality of Life in Women with Urinary Incontinence Treated by a Complete Pelvic Floor Rehabilitation Program (Biofeedback, Functional Electrical Stimulation, Pelvic Floor Muscles Exercises, and Vaginal Cones). J Sex Med. 2010;7(3):1200-8.

8. Da Silva L, Lopes MH. Urinary incontinence in women: reasons for not seeking treatment. Rev Esc Enferm USP. 2009;43(1):72-78.

9. Fischer-Sgrott FO, Manffra EF, Junior WFSB. Qualidade de vida de mulheres com bexiga hiperativa refratária tratadas com estimulação elétrica do nervo tibial posterior. Rev Bras Fisioter. 2009;13(6):480-6.
10. Berghmans LCM, Hendriks HJM, Bie RA, Doorn ESCVW, Kerrebroeck PHEV. Conservative treatment of urge urinary incontinence in women: a systematic review of randomized clinical trials. BJU Int. 2000;85:254-63.

11. Wang AC, Wang Y, Chen M. Single, randomized trial of pelvic floor muscle training, biofeedback-assisted pelvic floor muscle training, and electrical stimulation in the management of overactive bladder. Urology. 2004;63(1):61-66.

12. Bergmans LCM, Nieman F, van Waalwijk, van Doorn ESC. Effects of physiotherapy, using the adapted Dutch I-QOL in women with urinary incontinence (UUI). Int Urogynecol J Pelvic Floor Dysfunct. 2001;12(suppl 3):S40.

13. Okada N, Igawa Y, Nishizawa O. Functional electrical stimulation for detrusor instability. Int Urogynecol J. 1999;10:329-35.

14. Appell RA. Electrical stimulation for the treatment of urinary incontinence. Urology. 1998;51:24-26.

15. van Balken MR, Vandoninck V, Gisolf KWH, Vergunst H, Kiemeney LA, Debruyne FM, Bemelmans BL. Posterior tibial nerve stimulation as neuromodulative treatment of lower urinary tract dysfunction. J Urol. 2001;166(3):914-8.

16. Svihra J, Urca E, Luptak J, Kliment J. Neuromodulative treatment of overactive bladder noninvasive tibial nerve stimulation. Bratis। Lek Listy. 2002;103(12):480-3.

17. Amarenco G, Ismael SS, Schneider A, Raibaut P, Demaille-Wlodyka S, Parratte B, Kerdraon J. Urodynamic effect of acute transcutaneous posterior tibial nerve stimulation in overactive bladder. J Urol. 2003;169(6):2210-5.

18. Ciconelli RM. Translation and validation to the Portuguese of the Medical Outcomes Study 36 - Item Short-Form Health Survey (SF-36) [doctoral thesis]. São Paulo, Brazil: Federal University of São Paulo, 1997.

19. Patrick DL, Martin ML, Bushnell DM, Yalcin I, Wagner TH, Buesching DP. Quality of Life of Women with Urinary Incontinence: Further Development of the 


\section{Referências (cont.)}

Incontinence Quality of Life Instrument (I-QOL). Urology. 1999;53:71-6.

20. van Melick HHE, Gisolf KWH, Eckhardt MD, van Venrooij GEPM, Boon TA. One 24-hour frequency-volume chart in a woman with objective urinary motor urge incontinence is sufficient. Urology. 2001;58(2):188-92.

21. Lukacz ES, Lawrence JM, Burchette EJ, Luber KM, Nager CW, Buckealter JG. The use of visual analog scale in urogynecologic research: a psychometric evaluation. Am J Obstet Gynecol. 2004;19(1):165-70.

22. Zanetti MRD, Castro RA, Rotta AL, Santos PD, Sartori M, Girão MJBC. Impact of supervised physiotherapeutic pelvic floor exercises for treating female stress urinary incontinence. Sao Paulo Med J. 2007;125(5):265-9.

23. van der Pal F, van Balken MR, Heesakkers JPFA, Debruyne FMJ, Kiemeney LALM, Bemelmans BLH. Correlation between quality of life and voiding variables in patients treated with percutaneous tibial nerve stimulation. BJU Int. 2006;97:113-6.
24. Peters KM, Carrico DJ, Perez-Marrero RA, Khan AU, Wooldridge LS, Gregory L. Davis GL, MacDiarmid SA. Randomized Trial of Percutaneous Tibial Nerve Stimulation Versus Sham Efficacy in the Treatment of Overactive Bladder Syndrome: Results From the Sumit Trial. J Urol. 2010;183:1438-43.

25. Feldner PCJ, Sartori MGF, Lima GR, Baracat EC, Girão MJBC. Diagnóstico clínico e subsidiário da incontinência urinária. Rev Bras Ginecol e Obstet. 2006;28(1):54-62.

26. Vandoninck V, van Balken MR, Agro EF, Heesakkers JPFA, Debruyne FMJ, Kiemeney LALM, Bemelmans BLH. Posterior Tibial Nerve Stimulation in the Treatment of Voiding Dysfunction: Urodynamic Data. Neurourol Urodyn. 2004;23:246-251.

27. Stach-Lempinen B, Kujansuu E, Laippala P, MetsaÈnoja R. Visual analogue scale, urinary incontinence severity score and $15 \mathrm{~d}$-psychometric testing of three different health-related quality-of-life instruments for urinary incontinent women. Scand J Urol and Nephrol. 2001;35:476-483. 